\title{
Interactive Boundary Detection for Automatic Definition of 2D Opacity Transfer Function
}

\author{
Martin Rauberger, Heinrich Martin Overhoff \\ Medical Engineering Laboratory, University of Applied Sciences Gelsenkirchen, \\ Gelsenkirchen, Germany \\ martin.rauberger@fh-gelsenkirchen.de
}

\begin{abstract}
In computer assisted diagnostics nowadays, high-value 3-D visualization intake a supporting role to the traditional $2-\mathrm{D}$ slice wise visualization. 3-D visualization may create intuitive visual appearances of the spatial relations of anatomical structures, based upon transfer functions mapping data values to visual parameters, e.g. color or opacity. Manual definition of these transfer functions however requires expert knowledge and can be tedious. In this paper an approach to automatizing 2-D opacity transfer function definition is presented. Upon few parameters characterizing the image volume and an user-depicted area of interest, the procedure detects organ surfaces automatically, upon which transfer functions may automatically be defined. Parameter setting still requires experience about the imaging properties of modalities, and improper setting can cause falsely detected organ surfaces. Procedure tests with CT and MRI image volumes show, that real time structure detection is even possible for noisy image volumes.
\end{abstract}

\section{Introduction}

Direct volume rendering (DVR) of medical image volumes from modalities, like X-ray CT, MRI, 3-D ultrasound, or PET can often give a clear hint to morphologic, physiologic and/or functional pathologies. With the technological progress of imaging modalities producing image volumes of steadily increasing quality, 3$\mathrm{D}$ visualization techniques take in a viable supportive function to the traditional 2-D slice-wise finding in clinical routine.

DVR relies on opacity transfer functions (OTFs) to define the transparency (transparency $t$, opacity $\alpha=1-t$ ) of each voxel [1], and thus to visually separate the diagnostically relevant anatomical structures (structures of interest) from the dispensable structures. OTFs are however to be defined for every finding and image volume, requiring a definition process to be flexible for varying application purposes and time saving, in order to reach clinical acceptance.

\subsection{The projected histogram}

Kindlmann and Durkin [2] introduced that material boundaries are distinguishable as arches in a greyscale encoded frequency distribution in the domain of data 
value (voxel $\mathbf{x}$, data value $f(\mathbf{x})$ ) versus first order gradient magnitude $\left(\left|f^{\prime}(\mathbf{x})\right|\right.$ ), further referred to as projected histogram. Different types of homogeneous material $\left(v_{n}\right)$ appear as clotted regions (dark), whereas boundaries between these materials occur as regions of continuous data value change, and thus of higher gradient magnitude as seen in Fig. 1. In present medical imaging these curves are however not infinitely thin and regular, but blurred and distorted due to material inhomogeneities and characteristics of the imaging modality. By defining 2-D OTFs assigning highest opacity values to one boundary arch in the projected histogram, materials can be visually separated.

\subsection{Related work}

The topic of defining OTFs has been increasingly discussed in recent years. As the approach presented in this paper focuses on material boundaries and for 2-D OTF definition, only related work for visualizing material boundaries using 2-D OTFs is discussed.

Kniss et al. [3] proposed interaction widgets, with which to select peaks of the arches for 2-D OTF definition. Upon these widgets, a higher level of abstraction to alleviate user interaction is proposed by Salama et al. [4], where the number of freely definable parameters is cleverly reduced. But however to the cost of flexibility due to the specialization on one certain application.

Sereda et al. [5] address remaining insufficiency in the visual separation of 2-D OTFs using data value and gradient, introducing the L-H-histograms for improved separation of materials and their boundaries.

These approaches all succeed visual separation acceptable for clinical finding, but do however require the user to interact with additional elements to those of slice-by-slice viewing. Moreover do some propose direct interaction in an abstract projected histogram, requiring expert knowledge for OTF definition.

In order to avoid intense interaction with images and image-related parameters, a method for boundary detection based solely on interaction with the visualized image volume is presented. It exploits scatter plots and calculates
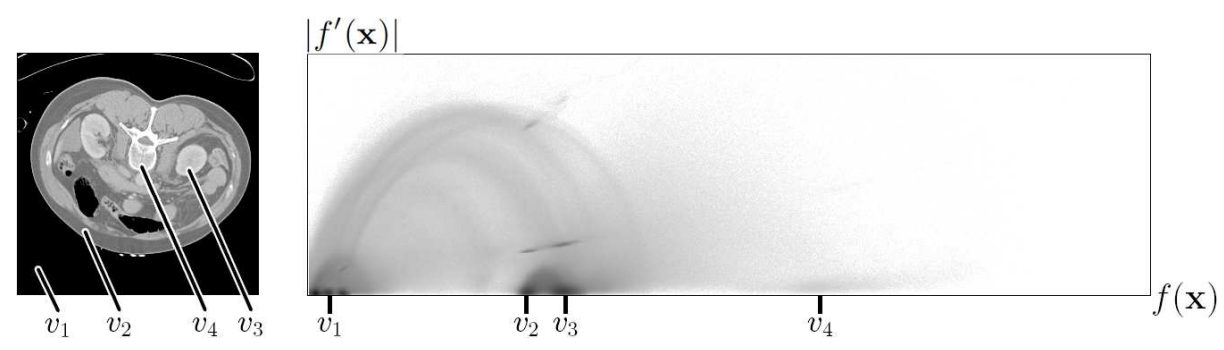

Fig. 1. Example of a projected histogram with first order gradient magnitude $\left|f^{\prime}(\mathbf{x})\right|$ versus grey-scale $f(\mathbf{x})$ (right) shown together with a slice of CT image volume of human torso (left). 
mathematical arch representations for later automatic 2-D OTF definition. Its performance is investigated with the focus on clinical application.

\section{Materials and methods}

Using a standard 2-D mouse as input device, the user can specify a structure of interest in the visualized image volume by e.g. clicking, resulting in a vector describing a straight path throughout the volume. By summing-up the data values and gradients of passed voxels, a scatter plot is calculated. Four examples of scatter plots are seen in Fig. 2, where four straight sampling paths visualize material boundary arches between aorta and mediastinum in a CT image of the human thorax. It is seen, that scatter plots of the same anatomical structure (aorta) vary throughout the image volume, due to the material inhomogeneities and imaging characteristics mentioned in chapter 1.1 .

In order to receive the best fitting geometric representation of the boundary arch within reasonable time, approximations of skewed circles are preferred due to their calculatory simplicity (in opposition to e.g. ellipses or splines). Approximations are performed upon numerous scatter plots collected by incorporating the local neighborhood of the passed voxels. For this instance the method of least squares is applied and its sum is compared to a predefined maximum value.

The approximation routine is initiated when a material boundary is supposed passed, by evaluating the gradient magnitudes mean value $\left(\overline{\left|f^{\prime}\right|}\right)$ and data value standard deviation $\left(\sigma_{f}\right)$ of every passed voxel and local neighborhood. For this
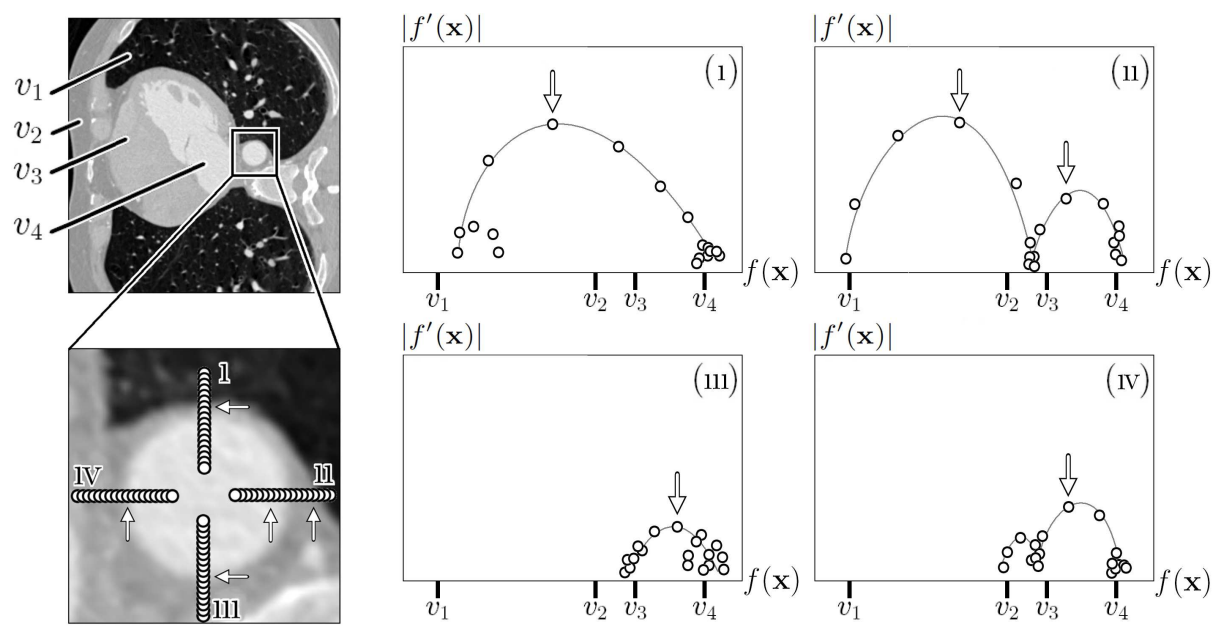

Fig. 2. Four examples of scatter plots calculated from straight sampling paths. Thin spline approximated arches depict material boundaries between aorta and mediastinum in a CT image of the human thorax. Thick lines depict the simple geometric representations of skewed circle approximations. 
instance, $\overline{\left|f^{\prime}\right|}$ and $\sigma_{f}$ are compared to predefined parameters determining the image volume.

After validating the relative position of the geometric representation to a predefined minimum gradient value $\left(\left|f^{\prime}\right|_{\min }\right)$, the resulting mathematical descriptions of material boundary arches in the projected histogram may then serve as background for automatic 2-D OTF definition.

\section{Results}

The presented approach succeeds in finding even subtle material boundaries, needing the user to simply click on the structure of interest while interacting with nothing more than the visualized 3-D image volume (Fig. 3). The approach saves time in clinical finding, as the time from user clicking to calculated mathematical representation of the boundary arch is below 0.5 seconds. The testing platform was a standard PC with Intel Pentium ${ }^{\mathrm{TM}}$ Core 2 Duo CPU $2 \times 2.40 \mathrm{GHz}, 2048 \mathrm{~GB}$ DDR2-RAM, a GeForce 8800 graphics card and Windows XP ${ }^{\mathrm{TM}}$ Professional. The testing image volume was a DICOM MRI image volume with $576 \times 576 \times 96$ voxels.

\section{Discussion}

The approach provides the user with a fast tool for finding material boundaries intuitively, and to calculate mathematical representations for later automatic 2D OTF definition. As the boundary detection relies on predefined reference parameters (for gradient magnitudes mean value $\overline{f^{\prime}}$, data value standard deviation $\sigma_{f} \mathrm{~s}$ ), improper setting of these parameters may result in detecting only the most prominent material boundaries, or in falsely detecting material inhomogeneities as material boundaries. The process of finding representative values
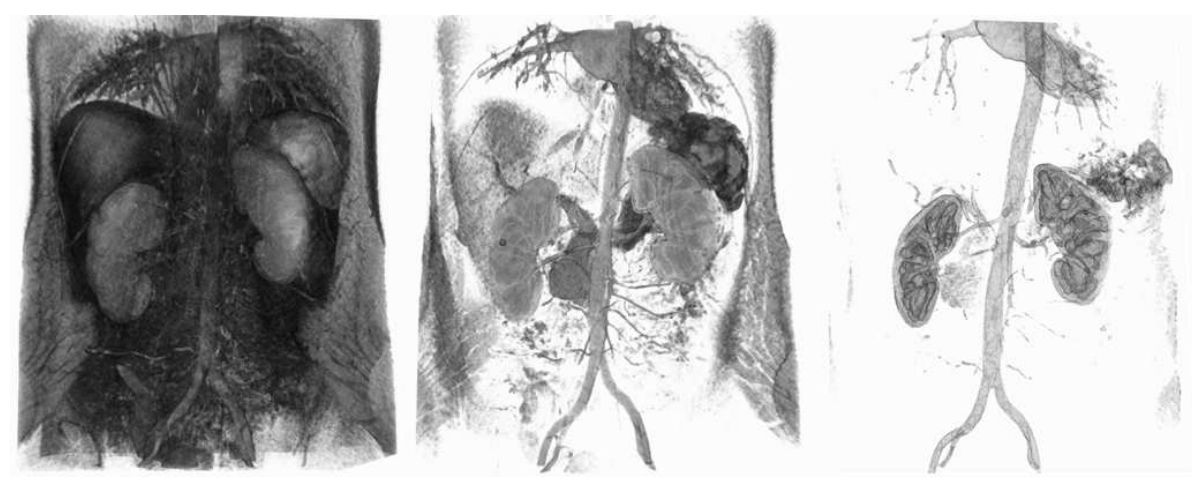

Fig. 3. Three examples of high-quality rendering results based upon a MRI image volume of the human abdomen. Through sequential clicking on an anatomical structure of interest (e.g. a kidney), the rendering becomes clearer from left to right. 
for these parameters may tedious and thus future research will focus on their automatic definition, through analysis and evaluation of the overall data value and gradient magnitude distribution.

Regardless of imaging modality, the data value ranges of anatomical structures vary throughout the image volume. As the mathematical representation calculated from a spatially narrowed region (scatter plot), the OTF may thus lack in visualizing the full extent of the structure of interest. The final rendering may thus only partially visualize anatomical structures, which can be misleading to interpret. Moreover may interfering structures be visualized, that share the same data value range, requiring expert knowledge in order to determine faulty and misleading visualization results. Solutions to this problem may be considered in applying a neighboring algorithm, only visualizing voxels within a solid structure around a seed point, or utilizing local transfer functions [6] applying several OTFs spatially separated throughout the image volume and thus compensating the spatial variances of the structure of interest. Also, introducing additional data value dimensions (e.g. $\mathrm{L}$ and $\mathrm{H}$ values [5]) may provide improved visual separation.

\section{References}

1. Levoy M. Display of surfaces from volume data. IEEE Computer Graph Appl. 1988;8(3):29-37.

2. Kindlmann GL, Durkin JW. Semi-automatic generation of transfer functions for direct volume rendering [Master's thesis]. Proc IEEE Symp Vol Vis. 1998; p. 79-86.

3. Kniss J, Kindlmann GL, Hansen CD. Multi-dimensional transfer functions for volume rendering. In: Johnson CR, Hansen CD, editors. The Visualization Handbook. Academic Press; 2004. p. 181-201.

4. Salama CR, Keller M, Kohlmann P. High-level user interfaces for transfer function design with semantics. IEEE Trans Vis Computer Graph. 2006;12(5):1021-1028.

5. Šereda P, Bartrolí AV, Serlie IWO, et al. Visualization of Boundaries in Volumetric Data Sets Using lh Histograms. In: IEEE Trans Vis Computer Graph. vol. 12; 2006.

6. Šereda P. Facilitating the Design of Multidimensional and Local Transfer Functions for Volume Visualization. Advanced School for Computing and Imaging. Delft, The Netherlands; 2007. 